Method of spatial size measurement of relativistic electrons beams with small bunch length

To cite this article: Yu.A. Goponov et al 2020 JINST 15 C04025

View the article online for updates and enhancements.

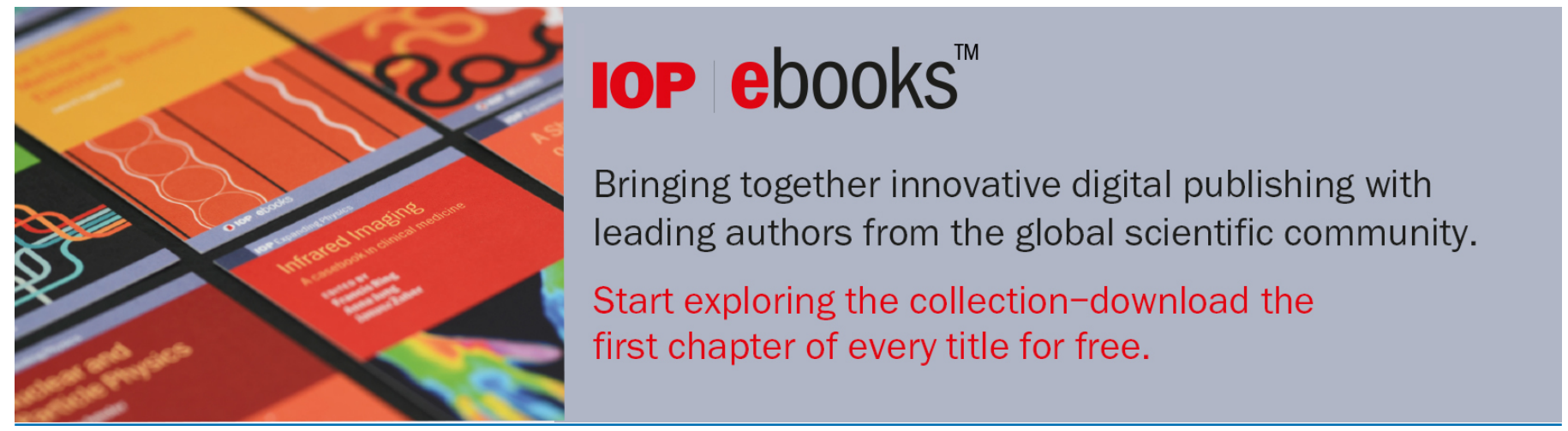


Xill International Symposium on Radiation from Relativistic Electrons in Periodic Structures - RREPS-19

SePteMBER 16-20, 2019

Belgorod, Russian Federation

\title{
Method of spatial size measurement of relativistic electrons beams with small bunch length
}

\author{
Yu.A. Goponov, ${ }^{a}$ R.A. Shatokhin, ${ }^{a}$ M.A. Sidnin, ${ }^{a}$ K. Sumitani, ${ }^{b}$ Y. Takabayashi, ${ }^{c}$ \\ I.E. Vnukov ${ }^{a, 1}$ and I.S. Volkov ${ }^{a}$ \\ ${ }^{a}$ Belgorod National Research University, \\ 85 Pobedy str., 308015 Belgorod, Russia \\ ${ }^{b}$ Japan Synchrotron Radiation Research Institute (JASRI), \\ 1-1-1 Kouto, Sayo-cho, Sayo-gun, Hyogo 679-5198, Japan \\ ${ }^{c}$ SAGA Light Source, Tosu, Saga 841-0005, Japan \\ E-mail: vnukov@bsu.edu.ru
}

ABSTRACT: We investigate the practical implementation of a previously proposed method for determining the transverse dimensions of an electron beam on a target by measuring the two-dimensional angular distributions of diffracted transition radiation of relativistic electrons for two distances between the crystal where the radiation is generated and the coordinate detector for femtosecond bunches. We show that determining electron microbunches with small longitudinal sizes requires an increased photon energy, achieved by decreasing the observation angle. The sensitivity limits of the method and the influence of the emission of secondary electrons and photons from the detector pixels are discussed.

KEYwORDS: Beam-line instrumentation (beam position and profile monitors; beam-intensity monitors; bunch length monitors); Detector modelling and simulations I (interaction of radiation with matter, interaction of photons with matter, interaction of hadrons with matter, etc); Instrumentation for FEL

\footnotetext{
${ }^{1}$ Corresponding author.
} 


\section{Contents}

1 Introduction 1

2 Method description $\quad 2$

3 Determination of the transverse dimensions of the electron beam 3

4 Conclusions $\quad 7$

\section{Introduction}

The divergence and transverse size of accelerated particles beams are important parameters for accelerators. In linear electron accelerators, optical radiation such as optical transition radiation (OTR) [1] and optical diffraction radiation (ODR) [2] is widely used to measure the beam parameters. However, it has recently been shown that OTR cannot be used to measure the profiles of electron beams of linear accelerators used to create free-electron x-ray lasers [3], since OTR becomes coherent if the beam longitudinal size becomes comparable with the wavelength of the observed radiation [4].

This coherence effect can be avoided by using radiation with a shorter wavelength, and the use of parametric x-ray radiation (PXR) has been proposed [5, 6]. PXR is quasimonochromatic, has a relatively narrow angular distribution, and emits at a large angle in the direction of the particle motion $\Theta_{D}=2 \Theta_{B}$, where $\Theta_{B}$ is the Bragg angle; see, for example, [7] and the literature cited therein. Measurements of the angular distributions of PXR using coordinate detectors $[6,8,9]$ have confirmed the feasibility of determining the beam size using this method.

In ref. [10], a calculation technique was proposed that takes into account the effect of the transverse dimensions of the particle beam on the radiation angular distribution for three mechanisms: PXR, diffracted bremsstrahlung (DB), and diffracted transition radiation (DTR). Comparisons of the calculation results with experimental data [11, 12] showed good agreement and confirmed that the method is satisfactory. In this article, a model-independent method is proposed for determining the size of an electron beam in the horizontal and vertical planes by measuring the two-dimensional angular distributions of radiation for two distances between the crystal and the coordinate detector.

In ref. [13], it was shown that the error in determining the beam sizes by the method of ref. [10] does not exceed 5-7\%, and the minimum measured dimensions $\sigma_{x, y}$ are determined by the conditions $\sigma_{x, y}>\delta$ and $\sigma_{x, y} / R>0.1 \Theta_{c h}$, where $\delta$ is the pixel size of the coordinate detector, $R$ is the distance between the crystal and detector, and $\Theta_{c h}$ is the characteristic radiation angle.

For particles of energy $E_{e}<1 \mathrm{GeV}$, the predominant mechanism of electron emission in crystals is PXR with a characteristic angle of $\Theta_{p h}=\sqrt{\gamma^{-2}+\omega_{p}^{2} / \omega^{2}}$ [7], where $\gamma$ is the Lorentz factor and $\omega$ and $\omega_{p}$ are the photon and plasmon energies of the material, respectively. The minimum measured dimensions are limited by the distance between the detector and the target and cannot be less than 
50-100 microns [13]. For electron energies above $5 \mathrm{GeV}$, the PXR contribution in the centre of the emission spot is negligible [14]. The highest angular density of DTR with a characteristic emission angle of $\Theta_{c h} \sim \gamma^{-1}$ reduces the minimum dimensions that can be determined using the method of ref. [10], which is mainly limited by the size of the element of the coordinate detector, with a minimum measured beam size of $\sigma_{x, y} \sim 10-15$ microns [13].

Reference [13] investigated the use of radiation with a photon energy of $\omega \sim 11 \mathrm{keV}$ and a wavelength of $\lambda \sim 0.1 \mathrm{~nm}$, which is comparable to the size of microbunches of the particle beam of an X-ray free-electron laser [3]. It is desirable to reduce the wavelength of the detected radiation to apply the technique of $[10,13]$ for FEL diagnostic purposes.

Based on the above, it is important and pertinent to study the possibilities of extending the method of ref. [10] for determining the transverse dimensions of electron beams, including X-ray free-electron lasers, and the limits of its applicability.

\section{Method description}

This section provides a brief description of how to estimate the beam size on a crystal following the method of refs. $[10,13]$. The effect of beam size and the distance between the crystal and the detector on the measured two-dimensional angular radiation distribution (see for example [6]) can be described by:

$$
Y\left(\theta_{y}, \theta_{x}\right)=\iint Y\left(\theta_{y}^{\prime}, \theta_{x}^{\prime}\right) G\left(\theta_{y}^{\prime} \rightarrow \theta_{y}, \theta_{x}^{\prime} \rightarrow \theta_{x}\right) d \theta_{y}^{\prime} d \theta_{x}^{\prime},
$$

where $\theta_{x}$ and $\theta_{y}$ are the horizontal and vertical angles of the photons, measured from the centre of the reflection angle $\Theta_{D}$ relative to the direction of the electron beam. $Y\left(\theta_{y}, \theta_{x}\right)$ and $Y\left(\theta_{y}^{\prime}, \theta_{x}^{\prime}\right)$ are the angular distributions of the radiation for an extended and a point-like beam of particles on the target, respectively, where the integration is carried out over a full solid angle. The function $G\left(\theta_{y}^{\prime} \rightarrow \theta_{y}, \theta_{x}^{\prime} \rightarrow \theta_{x}\right)$ describes the connection between the variables of each of these distributions. For a two-dimensional Gaussian distribution of the scatter of the points where the electrons hit the crystal, the function can be presented in the form:

$$
G\left(\theta_{y}^{\prime} \rightarrow \theta_{y}, \theta_{x}^{\prime} \rightarrow \theta_{x}\right)=\frac{1}{2 \pi \sigma_{y}^{\prime} \sigma_{x}^{\prime}} \exp \frac{-\left(\theta_{y}-\theta_{y}^{\prime}\right)^{2}}{2\left(\sigma_{y}^{\prime}\right)^{2}} \exp \frac{-\left(\theta_{x}-\theta_{x}^{\prime}\right)^{2}}{2\left(\sigma_{x}^{\prime}\right)^{2}}
$$

where $\sigma_{x}^{\prime}=\sigma_{x} / R$ and $\sigma_{y}^{\prime}=\sigma_{y} / R$ are the effective divergence angles in the horizontal and vertical planes, and $R$ is the distance between the crystal and the detector. The above expressions show that a change in the distance between the crystal and the detector changes the recorded angular distribution of the radiation, which can then be used to determine the beam size by comparing the distributions for different distances between the crystal and the detector.

For a detector placed at a distance $R$, the measured two-dimensional angular distribution of radiation intensity $Y_{R}\left(\theta_{y_{i}}, \theta_{x_{j}}\right)$ can be written in the following form:

$$
Y_{R}\left(\theta_{y_{i}}, \theta_{x_{j}}\right)=\iint_{\Delta \Omega\left(y_{i}, x_{j}\right)} Y_{R}\left(\theta_{y}, \theta_{x}\right) d \theta_{y} d \theta_{x},
$$

where $Y_{R}\left(\theta_{y}, \theta_{x}\right)$ is defined in accordance with expressions (2.1) and (2.2). $\Delta \Omega\left(y_{i}, x_{j}\right)$ is the solid angle covered by the coordinate detector element located at the point $y_{i}, x_{j}$. 
It is clear that the difference in the distributions $Y_{R_{1}}\left(\theta_{y_{i}}, \theta_{x_{j}}\right)$ and $Y_{R_{2}}\left(\theta_{y_{i}}, \theta_{x_{j}}\right)$, measured for distances $R_{1}$ and $R_{2}$, is due only to the characteristic dimensions of the beam and the distances between the crystal and the detector. The distributions are the results of convolutions of the angular distribution for a point-like electron beam of $Y\left(\theta_{y}^{\prime}, \theta_{x}^{\prime}\right)$ and two two-dimensional Gaussian distributions with standard deviations $\sigma_{x_{1}, y_{1}}^{\prime}=\sigma_{x, y} / R_{1}$ and $\sigma_{x_{2}, y_{2}}^{\prime}=\sigma_{x, y} / R_{2}$.

We assume that $R_{1}=k \cdot R_{2}$, where $k$ is an integer coefficient and the solid angles covered by the detectors are the same in each measurement, that is, the sizes of the detector elements for each of the distances differ by a factor of $k$. In this case, in the first approximation, we can assume that $Y_{R_{2}}\left(\theta_{y_{i}}, \theta_{x_{j}}\right)$ is a convolution of the distribution $Y_{R_{1}}\left(\theta_{y_{i}}, \theta_{x_{j}}\right)$ with a Gaussian distribution with a dispersion that depends on the unknown sizes of the beam on the target and on $R_{1}$ and $R_{2}$.

To determine the desired beam sizes on the target, we use the ordinary least squares method, minimizing the quadratic form:

$$
\begin{aligned}
\sum_{i=1}^{n} \sum_{j=1}^{m}\left(Y_{R_{2}}\left(\theta_{y_{i}}, \theta_{x_{j}}\right)-\frac{1}{2 \pi \widetilde{\sigma}_{x}^{\prime} \widetilde{\sigma}_{y}^{\prime}} \sum_{i^{\prime}=1}^{n} \sum_{j^{\prime}=1}^{m} Y_{R_{1}}\left(\theta_{y_{i^{\prime}}}, \theta_{x_{j^{\prime}}}\right)\right. \\
\left.\exp \left(-\frac{\left(\theta_{y_{i}}-\theta_{y_{i^{\prime}}}\right)^{2}}{2\left(\widetilde{\sigma}_{y}^{\prime}\right)^{2}}\right) \exp \left(-\frac{\left(\theta_{x_{j}}-\theta_{x_{j^{\prime}}}\right)^{2}}{2\left(\widetilde{\sigma}_{x}^{\prime}\right)^{2}}\right)\right)^{2}=\text { Min }
\end{aligned}
$$

where $m$ and $n$ are the numbers of points of the measured distributions in the horizontal and vertical directions and $\widetilde{\sigma}_{x}^{\prime}$ and $\widetilde{\sigma}_{y}^{\prime}$ are fitting parameters that minimize this form and are related to the size of the beam on the target $\widetilde{\sigma_{x}}, \widetilde{\sigma_{y}}$ as:

$$
\widetilde{\sigma}_{y, x} \approx \frac{k \cdot R_{2}}{\sqrt{k^{2}-1}} \widetilde{\sigma}_{y, x}^{\prime}
$$

\section{Determination of the transverse dimensions of the electron beam}

To test the capabilities of the technique and determine the limits of its applicability, a calculation was carried out to determine the parameters of an electron beam with an energy of $10 \mathrm{GeV}$ from two-dimensional distributions of diffracted transition radiation using a variation of the convolution parameters. The simulation was carried out for a reflection (022) of a silicon crystal and an observation angle of $32.2^{\circ}$. The detector element size was $10 \times 10 \mu \mathrm{m}^{2}$ for short distances and $20 \times 20 \mu \mathrm{m}^{2}$ for larger distances. The size of the electron beam on the crystal was $\sigma_{x}=40 \mu \mathrm{m}$ and $\sigma_{y}=20 \mu \mathrm{m}$. The plane on which the photons were reflected was vertical.

For the DTR angular distribution, we used a formula obtained in ref. [15]. To obtain the angular distribution of the radiation from a point-like electron beam $Y\left(\theta_{x}^{\prime}, \theta_{y}^{\prime}\right)$, the DTR angular distribution was convolved with a two-dimensional Gaussian distribution describing an electron beam with a divergence angle of $\theta_{\mathrm{e}}=15 \mu \mathrm{rad}$.

To determine the sensitivity of the method, we carried out a cycle of estimates of the beam size obtained using this technique for the same conditions, depending on the distance between the crystal and the detector. To take into account the possible influence of the statistical spread of the measurement results, noise with a uniform distribution in the range of $\pm 10 \%$ was added at each point to the dependences $Y_{R_{1}}\left(\theta_{y_{i}}, \theta_{x_{j}}\right)$ and $Y_{R_{2}}\left(\theta_{y_{i}}, \theta_{x_{j}}\right)$. In each fitting, the noise was factored in. The dependence of the estimated beam size $\widetilde{\sigma}_{y, x}$ on the distance was obtained from the fitting, 
shown in figure 1. The standard deviation of the resulting values from the mean was taken as the error. We assumed that $R_{1}=2 R_{2}$.

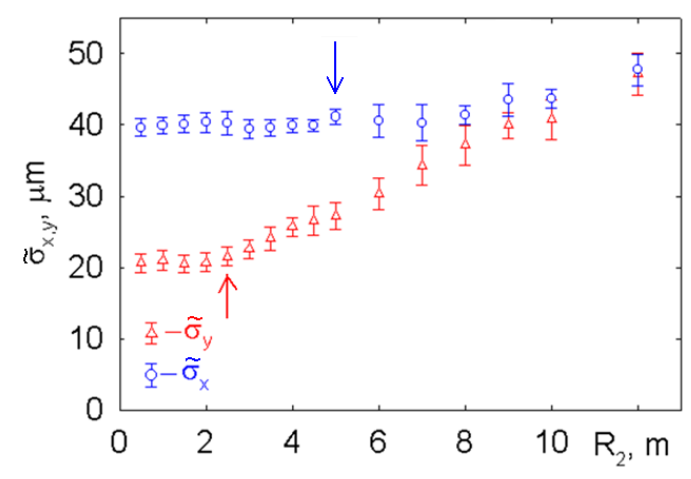

Figure 1. Dependence of the beam size estimates on the distance between the crystal and the detector. Silicon crystal, electron energy $10 \mathrm{GeV}$, observation angle $\Theta_{D}=32.2^{\circ}, \sigma_{x}=40 \mu \mathrm{m}, \sigma_{y}=20 \mu \mathrm{m}$.

Figure 1 shows that for distances $R_{2}$ less than two and a half meters, the error in determining the vertical size of the beam does not exceed $6 \%$, and the values obtained from the fitting coincide with those in the simulation. For large distances, the difference between the estimate $\widetilde{\sigma}_{y}$ and the beam size of $\sigma_{y}=20$ microns increases. At the same time, the fitting error grows. For the horizontal beam size, deviation and growth of the fitting error begin for distances greater than $5 \mathrm{~m}$. The arrows show the boundaries of the area of applicability of the technique for a noise level of $\pm 10 \%$. In both cases, the differences begin to exceed the fitting error when $\sigma_{x, y}^{\prime}=\sigma_{x, y} / R_{2} \leq 0.1 \gamma^{-1}$.

To understand the source of the errors in estimating $\widetilde{\sigma}_{x, y}$ and its spread, figure 2 shows the vertical angular distributions for a point-like and an extended electron beam for $R_{2}=2 \mathrm{~m}$ and $4 \mathrm{~m}$.
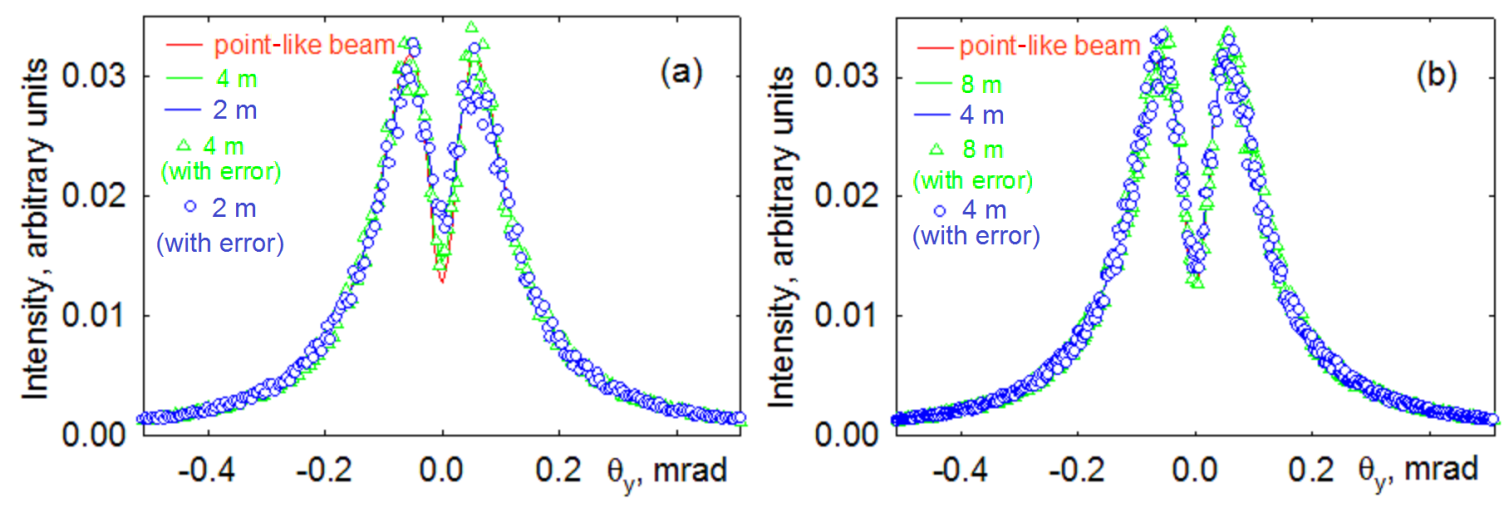

Figure 2. Vertical angular distribution of radiation, showing the radiation distribution for a point-like electron beam and distributions for extended electron beams with distances of $R_{1}=4 \mathrm{~m}, R_{2}=2 \mathrm{~m}$ (a) and $R_{1}=8 \mathrm{~m}, R_{2}=4 \mathrm{~m}$ (b). Electron energy $10 \mathrm{GeV}$, silicon crystal thickness 20 microns, (022) reflection order, observation angle $\Theta_{D}=32.2^{\circ}$.

Comparing figures $2 \mathrm{a}$ and $2 \mathrm{~b}$ for $R_{2}=2 \mathrm{~m}$ and $4 \mathrm{~m}$, it is clear that with increasing $R_{2}$, the differences between the distributions for different distances decrease and become almost invisible for $R_{2}=4 \mathrm{~m}$, and therefore the method loses sensitivity. Due to the larger horizontal size of the 
beam, an increase in the distance between the crystal and the coordinate detector has a weaker effect on the fitting error and the estimation value. It should be noted here that with an increase in the level of noise, a deviation of $\widetilde{\sigma}_{x, y}$ from $\sigma_{x, y}$ begins to appear for shorter distances.

Free-electron lasers in the X-ray frequency range [3] operate up to wavelengths of $\sim 0.1 \mathrm{~nm}$, which corresponds to a photon energy of $\omega \sim 15 \mathrm{keV}$. In other words, the wavelength we have chosen here and in ref. [13] to test the applicability and conditions of the method is comparable to the longitudinal dimensions of the bunch, which is insufficient to exclude coherent effects in the radiation. However, for $\omega \ll \gamma \omega_{p}$, which is valid for the X-ray frequency range, and an electron energy of $E_{e}>5 \mathrm{GeV}$, the angular distribution and intensity of the transition radiation, and consequently the DTR, are virtually independent of the photon energy [7]. Therefore, all the results and conclusions obtained above and in ref. [13] remain valid for shorter wavelengths. Furthermore, a decrease in the radiation wavelength by a factor of more than 10 to $\lambda \sim 0.01 \mathrm{~nm}$ is achievable, since in the experiment in ref. [16] for a diamond crystal and an observation angle of $4^{\circ}$, PXR and DB were clearly detected with a photon energy of $\omega \sim 145 \mathrm{keV}$.

The transverse dimensions of the electron beam for X-ray FEL implementation are of the order of tens of microns and higher, which allows the distance between the crystal and the coordinate detector to be increased without violating the condition $\sigma_{x, y}^{\prime}=\sigma_{x, y} / R_{2}>0.1 \gamma^{-1}$. This allows the photon energy to be increased by reducing the viewing angle while maintaining the ability to protect the detector from bremsstrahlung from the crystal; see ref. [13] for details.

Effect of emission of secondary electrons and quanta. The main condition for implementing the method to evaluate the beam size $[10,13]$ is that the solid angles covered by the detectors for both distances should be the same, provided by a proportional increase in the number of pixels for a greater distance. The emission of secondary electrons and quanta from the element, in which photons interact with the substances of the detector, in the neighbouring pixels distorts the measured distributions, smoothing them out. This effect is stronger for a smaller distance between the crystal and the detector due the smaller size of the detector element. Therefore, it is similar to the effect of increasing the beam size [13].

To verify the significance of the effect of the emission of secondary electrons and quanta, we performed a simulation of the methodology of $[10,13]$, with and without taking into account this effect, via a Monte Carlo approach, for the following conditions: electron energy $10 \mathrm{GeV}$, photon energy $\omega=15 \mathrm{keV}$, detector pixel sizes $10 \times 10 \mu \mathrm{m}^{2}$ and $20 \times 20 \mu \mathrm{m}^{2}$ for distances between the crystal and detector of $1 \mathrm{~m}$ and $2 \mathrm{~m}$, respectively. The characteristic sizes of the electron beam on the target were 10 microns and 20 microns in the horizontal and vertical planes, respectively. The divergence of an axially symmetric electron beam was $10 \mu \mathrm{rad}$.

To simplify the simulation process, instead of using the DTR angular distribution obtained in ref. [15], we used the azimuthally symmetric spectral-angular distribution of transition radiation, known as the Garibyan formula [7].

The simulation was carried out for P43 phosphor with chemical composition $\mathrm{Gd}_{2} \mathrm{O}_{2} \mathrm{~S}$ as the working substance of the HR25 detector [17] used in the experiment [8]. The phosphor plate has a thickness of 30 microns. The key features of the modelling technique are briefly described in [18]. A more detailed description of the modelling process and results, as well as the dependence of the efficiency and spatial resolution on the photon energy and phosphor thickness, will be given later. 
Figure 3 shows the ratio of the radiation intensities recorded by the detector for two distances in the horizontal (a) and vertical (b) planes. The simulation was carried out for the following conditions: a point-like electron beam (line) and an extended electron beam (circles) without taking into account the emission of secondary radiation, and a point-like electron beam taking into account the emission of secondary radiation (triangles).
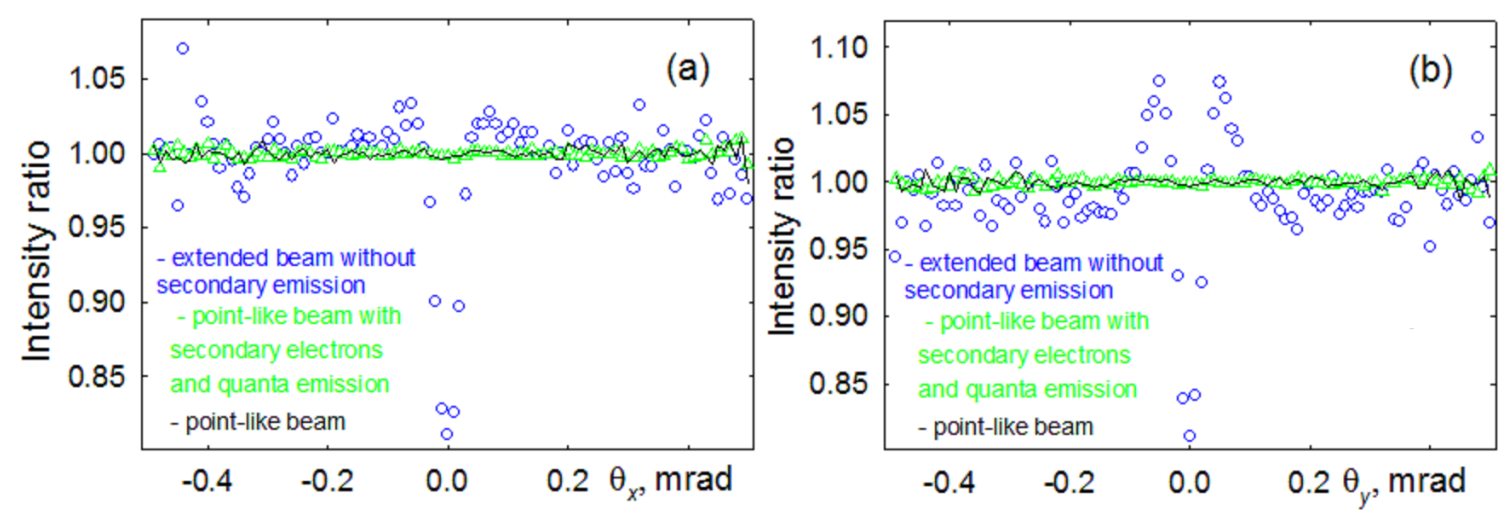

Figure 3. Ratio of radiation intensities for distant and close locations of the detectors in the horizontal (a) and vertical (b) planes.

Figure 3 shows that without taking into account the size of the electron beam on the target and the effect of the emission of secondary particles and quanta, the angular distributions measured for the two distances coincide. Their ratio is close to unity for the entire range of viewing angles. The scatter of values for large viewing angles is due to the low statistics arising from the sharp decrease in the transition radiation intensity with an increase in the photon emission angle (see figure 2). The influence of the process of secondary electrons and quanta emission (triangles in figure 3) is practically negligible because of their small path for a detected photon energy of $15 \mathrm{keV}$.

The effect of the size of the beam on the crystal (circles in figure 3) is more significant. The deviation of the ratio of radiation intensities for the two distances from unity reaches $20 \%$. The sizes of the electron beam in the vertical and horizontal planes differ, and therefore the ratios of the intensities recorded by the detector for the two distances differ in the vertical and horizontal planes, which is the basis for the method for determining the size of the electron beam in ref. [10]. Simultaneously considering the size of the beam on the target and the effect of the emission of secondary electrons and quanta did not practically change the ratio of radiation intensities for different distances compared with the situation where the emission of secondary electrons and quanta was not taken into account; therefore, this dependence is not presented.

Increasing the energy of the detected radiation to eliminate the effects of coherence in the radiation decreases the registration efficiency and increases the energy of secondary electrons and quanta. Modelling shows that for a phosphor thickness of 30 microns the registration efficiency changes from $\epsilon=54 \%$ for $\omega=15 \mathrm{keV}$ to $3 \%$ and $4.5 \%$ for photon energies of $50 \mathrm{keV}$ and $80 \mathrm{keV}$. The detector thickness increase leads to an increase in the registration efficiency but can increase the influence of the secondary emission, which should be monitored.

Figure 4 shows the ratio of the radiation intensities in the vertical plane recorded by the detector for two distances and for detector thicknesses of $100 \mu \mathrm{m}$ (a) and $200 \mu \mathrm{m}$ (b) for a photon energy of 
$50 \mathrm{keV}$. The simulation was carried out for the following conditions: a point-like electron beam without taking into account the emission of secondary radiation (line), a point-like electron beam (triangles) and an extended electron beam (circles) taking into account secondary emission.
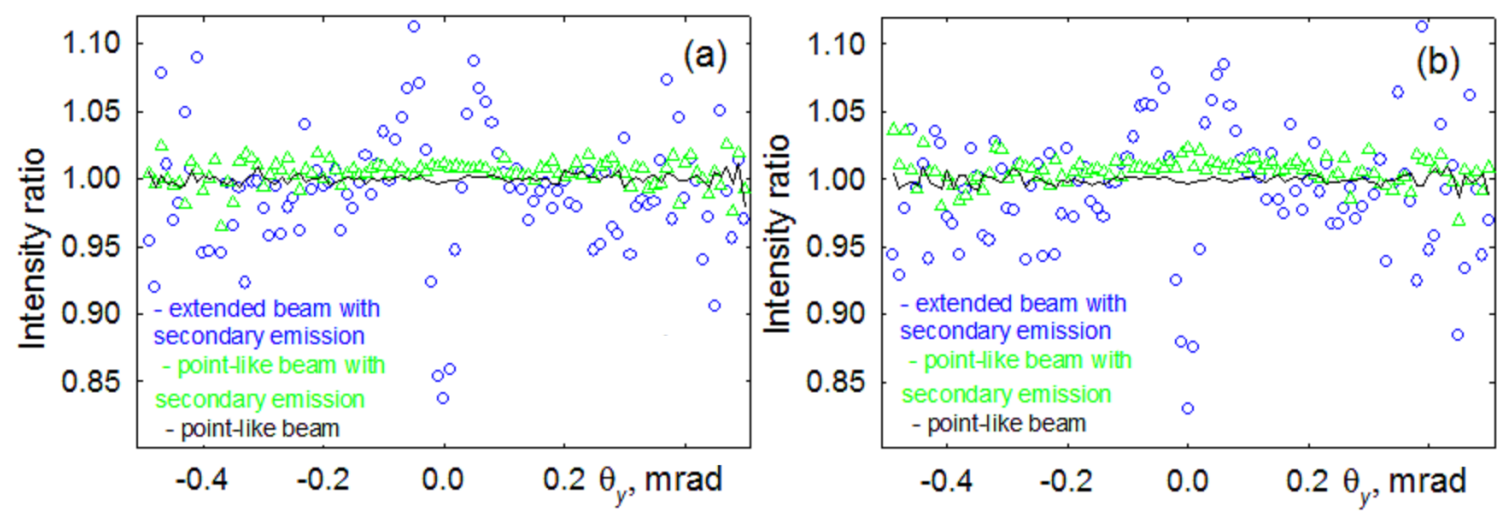

Figure 4. Ratio of radiation intensities for a photon energy of $50 \mathrm{keV}$ in the vertical plane for distant and close locations for detectors thicknesses of $100 \mu \mathrm{m}$ (a) and $200 \mu \mathrm{m}$ (b).

Figure 4 shows that the increase in photon energy had practically no effect on the ratio of intensities for the near and distant detector positions and the extended electron beam (circles) (see figures $3 \mathrm{~b}$ and 4 ), which confirms the assertion that the method applicability is independent of the photon energy. Also, the ratio of intensities for a point-like beam, taking into account the emission of secondary electrons and quanta (triangles), differs from unity. For a thicker detector (figure 4b), this difference is larger, in contrast to the ratio for an extended beam, which has not changed. The same behaviour has been observed for a photon energy of $80 \mathrm{keV}$.

The reason for the observed difference is the greater average path length of secondary electrons in a larger pixel and the consequent greater loss of energy in that pixel. As a result, the energy left by the detected radiation in the far detector for the entire range of viewing angles is $0.5-1.5 \%$ greater than in the near detector. This difference is significantly smaller than the contribution of the beam sizes on the target and, to a first approximation, need not be taken into account.

\section{Conclusions}

The size of an electron beam with energies above $5 \mathrm{GeV} \sigma_{x, y}$ can be determined by measuring the angular distributions of diffracted transition radiation of fast electrons in thin crystals for two distances that differ by at least a factor of two, between the source and the coordinate detector. The desired beam sizes are determined by fitting the distribution for the smaller distance by convolving the distribution for the larger distance with a two-dimensional Gaussian distribution, the parameters of which are uniquely related to the beam size and the distances between the crystal and the detectors.

The technique can determine the size of a beam with a small bunch length of the order of $0.1 \mathrm{~nm}$ or less by switching to a lower viewing angle and a higher photon energy up to $100-150 \mathrm{keV}$. The effect of the emission of secondary electrons and photons is small and can be neglected in the first approximation. 


\section{Acknowledgments}

This work was supported in part by JSPS KAKENHI Grant Number JP26400304.

\section{References}

[1] R.B. Fiorito, Recent Developments in Optical Transition Radiation Beam Diagnostics, Proceedings of PAC09 (2009) 741.

[2] J. Urakawa et al., Feasibility of optical diffraction radiation for a non-invasive low-emittance beam diagnostics, Nucl. Instrum. Meth. A 472 (2001) 309.

[3] The European X-Ray Free-Electron Laser Technical Design Report, DESY 2006-097, (2007).

[4] H. Loos et al., Observation of Coherent Optical Transition Radiation in the LCLS, proceedings of the International Free Electron Laser Conference FEL08, Republic of Korea, 24-29 August 2008, pg. 485.

[5] A. Gogolev, A. Potylitsyn and G. Kube, A possibility of transverse beam size diagnostics using parametric X-ray radiation, J. Phys. Conf. Ser. 357 (2012) 012018.

[6] Y. Takabayashi, Parametric X-ray radiation as a beam size monitor, Phys. Lett. A 376 (2012) 2408.

[7] P. Rullhusen, X. Artru and P. Dhez, Novel Radiation Sources Using Relativistic Electrons, World Scientific, Singapore, (1999) [DOI].

[8] G. Kube et al., Investigation of the applicability of parametric x-ray radiation for transverse beam profile diagnostics, proceedings of the 4th International Particle Accelerator Conference IPAC2013, Shanghai, China, 12-17 May 2013,pg. 491.

[9] Y. Takabayashi and K. Sumitani, New method for measuring beam profiles using a parametric $x$-ray pinhole camera, Phys. Lett. A 377 (2013) 2577.

[10] I.E. Vnukov, Y.A. Goponov, M.A. Sidnin, R.A. Shatokhin, K. Sumitani and Y. Takabayashi, Measuring the transverse sizes of an electron beam by the angular distribution of the coherent radiation of electrons in a crystal, J. Surf. Invest. 13 (2019) 515.

[11] Y. Takabayashi and A.V. Shchagin, Observation of parametric X-ray radiation by an imaging plate, Nucl. Instrum. Meth. B 278 (2012) 78.

[12] Y. Takabayashi, K. Korotchenko, Y. Pivovarov and T. Tukhfatullin, Angular distributions of parametric x-ray radiation from a diamond crystal, Nucl. Instrum. Meth. B 402 (2017) 79.

[13] Yu. A. Goponov et al., New method of electron beam transverse size measurement by angular distribution of emission in a thin crystal, Phys. Rev. Accel. Beams 22 (2019) 082803.

[14] Yu. A. Goponov, S.A. Laktionova, O.O. Pligina, M.A. Sidnin and I.E. Vnukov, Influence of real photon diffraction on parametric $X$-ray radiation angular distribution in thin perfect crystals, Nucl. Instrum. Meth. B 355 (2015) 150.

[15] A.P. Potylitsin and V.A. Verzilov, Parametric X-rays and transition-diffracted radiation in crystal stacks, Phys. Lett. A 209 (1995) 380.

[16] A.N. Baldin et al., About real photon diffraction contribution into measured PXR spectrum in perfect crystals (in Russian), J. Surf. Invest. 4 (2006) 72.

[17] High-Resolution X-Ray Camera, http://www.proxivision.de/datasheets/X-Ray-Camera-HR25-X-ray-PR-0055E-03.pdf.

[18] Yu. A. Goponov et al., Spatial distribution of PXR generated by $855 \mathrm{MeV}$ electrons: Comparison of simulation results with experimental data, Nucl. Instrum. Meth. B 402 (2017) 83. 